\title{
CLINICAL DIAGNOSIS AND TREATMENT OF SALIVARY GLANDS INFLAMMATION
}

\author{
Andreea TOMA ${ }^{1,2}$, Patricia FURDUI ${ }^{1}$, Alexandra CHIRILA ${ }^{1}$, Alina L.A. OANCEA ${ }^{1,2} \bowtie$, \\ Raluca GRIGORE ${ }^{1,2}$, Serban V.G. BERTESTEANU ${ }^{1,2}$ \\ ${ }^{1}$ ENT Department, Clinical Hospital Coltea, Bucharest, Romania \\ 2 University of Medicine and Pharmacy „Carol Davila“, Bucharest, Romania
}

Received 24 Oct 2019, Accepted 19 Nov 2019

https://doi.org/10.31688/ABMU.2019.54.4.14

\begin{abstract}
Inflammation of the salivary glands manifest differently, depending on the etiologic agent and patient population. Inflammatory and infectious pathology of the salivary glands is multifactorial and has a multimodal therapy. Bacterial infections are the result of ductal blockage or decreased salivary production. On this background, retrograde contamination of glandular parenchyma occurs. Some systemic viral infections produce salivary pathology such as mumps or AIDS. Many granulomatous infections can affect salivary glands such as tuberculosis (TB), syphilis, tularemia, toxoplasmosis, actinomycosis, and bartonellosis. Salivary inflammation occurs either in the secretory canal or in the whole gland. Suppuration of the salivary glands in immunosuppressed individuals can extend to cervicofacial regions and the mediastinum and require immediate treatment. Interdisciplinary collaboration with the infectionist, parasitologist and radiologist is the golden standard towards the benefit of the patient. Authors describe the clinical features and treatment of inflammatory diseases of the salivary glands.
\end{abstract}

Keywords: salivary glands, salivary lithiasis, mumps, AIDS.

\section{Résumé}

Diagnostic clinique et traitement de l'inflammation des glandes salivaires

L'inflammation des glandes salivaires se manifeste différemment selon l'agent étiologique et la population de patients. La pathologie inflammatoire et infectieuse des glandes salivaires est multifactorielle et comporte une thérapie multimodale. Les infections bactériennes sont le résultat d'un blocage canalaire ou d'une diminution de la production salivaire. Sur ce fond se produit une contamination rétrograde du parenchyme glandulaire. Certaines infections virales systémiques entrainent une pathologie salivaire telle que les oreillons ou le SIDA. De nombreuses infections granulomateuses peuvent toucher les glandes salivaires telles que la tuberculose, la syphilis, la tularémie, la toxoplasmose, l'actinomycose, la bartonellose. L'inflammation salivaire se produit soit dans le canal de sécrétion, soit dans toute la glande. La suppuration des glandes salivaires chez les personnes immunodéprimées peut s'étendre aux régions cervico-faciales et au médiastin et nécessiter un traitement immédiat. La collaboration interdisciplinaire avec l'infectionniste, le parasitologue et le radiologue est le standard d'or au bénéfice du patient. Les auteurs ont décrit les caractéristiques 


\section{List of abbreviations:}

AST $=$ antimicrobial susceptibility testing

$\mathrm{TB}=$ tuberculosis

$\mathrm{FNA}=$ fine needle aspiration

\section{INTRODUCTION}

There are different etiological agents involved in salivary glands inflammation that produce various forms. Bacterial infections are the result of ductal blockage or decreased salivary production. On this background, retrograde contamination of glandular parenchyma occurs. Some systemic viral infections produce salivary pathology such as mumps or AIDS. Many granulomatous infections can affect salivary glands such as TB, syphilis, tularemia, toxoplasmosis, actinomycosis, and bartonellosis. Salivary inflammation occurs either in the secretory canal or in the whole gland. The duct system is free of germs except the ductal papilla. The infection of the gland occurs most frequent through the canalicular pathway, through the lymphatic system from the nearby ganglia, through hematogenous dissemination, which is rare, or directly through wounds, otomastoiditis or mandibular osteitis. Contributing factors in salivary glands inflammation are: oral microbial exacerbation (lack of oral hygiene), salivary flow blockage (salivary lithiasis), decreasing canalicular motility (lack of mastication), hormonal disorder, metabolic disorder, immunosuppression, dehydration syndrome ${ }^{1}$.

\section{Non-specific bacterial acute sialadenitis}

It occurs in any salivary gland, but more frequent in one parotid gland, in men with an average age of 50-60 years ${ }^{2}$. Postoperative parotitis has an incidence of $0.01-0.02 \%$ in hospitalized patients and $0.002-0.04 \%$ in the postoperative period ${ }^{3}$. The germs involved are Staphylococcus aureus, Streptococcus spp., Gram-negative bacilli and anaerobic germs in immunosuppressed patients ${ }^{4}$.

There are three types of non-specific bacterial acute sialadenitis: catarrhal, purulent and gangrenous. The catarrhal form is characterized by an insidious onset, glandular hyposalivation, glandular swelling and spontaneous pain or on palpation. Through the ductal opening drains viscid saliva and fibrin clots. It evolves toward healing or to gangrenous form in three days ${ }^{5}$. The purulent form includes alteration of the general status, trismus, fever, rigors, high glandular swelling, acute pain with otic and/ or temporal irradiation, tegument congestion, and increased consistency. On gland palpation, soreness draining occurs that could lead to parotid or cliniques et le traitement des maladies inflammatoires des glandes salivaires.

Mots-clés: glandes salivaires, lithiase salivaire, oreillons, SIDA.

submandibular abscess. The gangrenous form is rare, occurs in immunosuppressed patients, with skin redness, tegument necrosis, palpable crepitus and facial paralysis. It spreads to the surrounding structures and it can lead to septic shock or toxic shock syndrome. A bacteriological examination of the salivary secretion should be done. The treatment implies broad-spectrum antibiotics, parenteral hydration, oral hygiene, sialagogues, anti-inflammatory drugs, external, bimanual massage for drainage or surgery in case of medical treatment failure. The antibiotics consists in penicillin with beta-lactamase inhibitor, first generation cephalosporins, clindamycin or vancomycin in case of penicillin allergies, steroidal and non-steroidal anti-inflammatory drugs, pilocarpine and Jaborandi tincture as sialagogues ${ }^{6}$. The duct system lavage is performed with intracanalicular instillation of normal saline, antibiotics and disinfectant solutions (high risk of intraparenchymal spreading of infection). The surgical treatment applies in case of local abscess that does not respond to medical treatment and consists in incision and draining. The differential diagnosis includes non-parotid edema, lymphangitis from external otitis, cervical adenitis, sebaceous cysts, dental abscess, mastoid Bezold's abscess, branchial cleft cysts and lymphoma. The complications of non-specific bacterial acute sialadenitis are disseminated suppuration, osteomyelitis, jugular vein thrombophlebitis, arterial bleeding, facial paralysis, respiratory obstruction, sepsis and death.

Particular forms of non-specific bacterial acute sialadenitis:

1. Neonatal suppurative parotitis may appear in premature infants, frequently in boys. The germs involved are Staphylococcus aureus, Escherichia coli, Pseudomonas aeruginosa, Group A $\beta$-hemolytic streptococcus? ${ }^{\text {? }}$ The oral cavity and the hematogenous pathway represent infection pathways. Present signs and symptoms are fever, irritability, anorexia, weight loss, edema, overlying erythema, fluctuating and hardened gland. A bacteriological exam from the duct system and from the salivary gland is needed to be done with fine needle aspiration (FNA). As for treatment, parenteral antibiotics against Streptococcus aureus and Gram-negative bacilli, adjustment of the antibiotics after antimicrobial susceptibility testing (AST). In case of fluctuating 
gland or volume increasing, despite the medical treatment, draining procedures are needed ${ }^{8}$.

2. Juvenile recurrent parotitis is rare, with unknown etiology and it frequently occurs in boys with a history of mumps. Recurrent gland impairment is present, usually accompanied by postprandial painful gland swelling 9 . Recommended treatment consists in antibiotics, canalicular lavage and parotidectomy only in case of medical treatment failure because of the risk of facial paralysis ${ }^{10}$.

\section{Chronic sialadenitis}

Chronic sialadenitis is characterized by local impairment of the salivary gland, repeated episodes of pain, inflammation, and parenchymal degeneration. The patients have a history of acute sialadenitis. The predisposing factors are ductal obstruction (ductal stones, strictures, mucus, papilla lesions, extrinsic compression), ductal dilatation, decreasing salivary secretion. The parotid gland is the most affected ${ }^{11}$. Recurrent episodes of swelling, pain, thickening and decreasing salivary secretion occur. The onset is insidious and the tegument appears to be normal or moderately infiltrated. It can be confused with a tumor due to lack of pain and increased consistency. The treatment consists in antibiotics, canalicular instillation (with blunt needle, for 5-10 minutes, with $2-3 \mathrm{ml}$ of hydrocortisone hemisuccinate, proteolytic enzymes, antibiotics, 6-8 instillations) ${ }^{12}$. The surgical treatment (surgical removal of the gland) is required only in case of medical treatment failure. None of the treatments are fully satisfactory ${ }^{13}$.

\section{Sialolithiasis}

Represents half of salivary gland diseases and it consists of intraluminal hard deposits in the salivary gland. It is often associated with chronic sialadenitis. The presence of the stone is responsible for the recurrent episodes. It occurs often in men of 50-60 years of age but it has been reported in children as well ${ }^{14}$. The predisposing factors are salivary stasis, ductal inflammation, ductal trauma and general causes. Sialolithiasis produces chronical inflammation and vice versa. The stones are made of calcium, phosphates, magnesium, zinc, glycoproteins, and mucopolysaccharides, but there is no correlation with blood calcium or phosphates levels. In all of the salivary glands, the development of the stones takes place in the hilum and it expands in the entire ductal system. Most often, it occurs in the submandibular gland (80\%), followed by the parotid gland (19\%) and the sublingual gland $(1 \%)^{15}$. The stones are yellow, rough, elongated, with a size between $1 \mathrm{~mm}$ to a few $\mathrm{cm}^{16}$.

1. Submandibular sialolithiasis can be caused by increased mucus secretion, alkaline, viscid, with high levels of calcium, phosphates, modified position of the gland, Wharton duct with two anatomical curves (on the posterior edge of the mylohyoid muscle or to the place where it crosses the lingual nerve), narrow orifice of Stenon canal and germs stagnation in the oral cavity. The clinical forms are represented by salivary colic, salivary abscess and "salivary tumor". In case of salivary colic, postprandial painful swelling is present with variable resolutions between attacks. In repetitive episodes, ductal stricture and parenchymal atrophy may appear. Sometimes stone traumas can lead to fistulas. They can be spontaneously removed under the pressure of the saliva or by complications with the occurrence of other clinical forms. The salivary abscess is a result of saliva retention caused by the stone. If the stone is above the mylohyoid muscle, it determines the inflammation of the Wharton duct, sublingual abscess, intense sublingual pain, pain radiating to the ear, hypersalivation, pain on tongue movement, oral floor swelling ("roaster comb"), thickened Wharton canal. If the stone is intraglandular or under the ductal mylohyoid part, it determines encapsulated intraglandular suppuration, painful pressure on the gland, blurred oral clinical signs. In evolution, submandibular abscess occurs. In „salivary tumor" (chronic sialadenitis), the subjective clinical signs can be absent or minimal. The objective clinical signs are represented by oversized, endured and irregular gland that can give a pseudo tumoral aspect. Prominent ostium and purulent discharge when pressing the gland can also be present.

2. In parotid sialolithiasis, the stone is usually present in the Stenon duct. It can have a staghorn appearance, with long latency period and rare salivary cramps. Often, chronic recurrent parotitis, can lead to pseudo tumoral sclerosis.

3. Sublingual sialolithiasis is rare and confused with the inflammation of the Wharton duct. The submandibular gland is normal.

4. Minor salivary gland sialolithiasis is extremely rare. The painful swelling is reduced. The removal of the stone can be spontaneous or through incision.

Clinical diagnosis of sialolithiasis is based on the medical history and bimanual palpation, which is difficult on parotid gland because of the small stones and the thickness of the cheek. A modern method of diagnosis is represented by intracanalicular endoscopy. The treatment for sialolithiasis depends on the location, size, number and stage of the stone. It can be medical, with antibiotics, anti-inflammatories, sialagogues and antiseptics. Repeated canalicular dilatation catheterization with normal saline, that eliminates the stone can also be used. The surgical 
treatment consists in stone ablation, submaxillectomy, parotidectomy, interventional sialendoscopy and lithotripsy. The stone ablation is performed when the glandular modifications are reversible. Under local oral anesthesia, papillotomy and duct incision are done. Possible complications are lingual nerve injury (especially in posterior locations) and strictures. Wide incision on Stenon duct is not recommended due to strictures. Submaxillectomy is indicated in case of retention of a glandular or hilum stone, stones bigger than $8 \mathrm{~mm}$, more than two, frequent relapse, lithotripsy failure and "salivary tumor" ${ }^{\text {"17 }}$. Parotidectomy is rarely indicated when the stone is superficial (superficial parotidectomy). It is extremely rare performed $(<5 \%)$ in case of multiple intraglandular stones or other treatments failure ${ }^{14,18}$. Complications are represented by facial nerve injury in glandular fibrous lesions and gland atrophy induction in Stenon ligature for deep stones. Interventional sialendoscopy consists in endoscopic catheterization of the salivary canal followed by the insertion of a special stone extraction device. It is indicated for stones smaller than $4 \mathrm{~mm}$ on Wharton canal and for stones smaller than $3 \mathrm{~mm}$ on Stenon canal ${ }^{19}$. A temporary stent is inserted to maintain and increase the caliber. The success rate is above $80 \%^{20}$. For larger stones, lithotripsy is used. Lithotripsy can be extracorporeal. Through pretherapeutic ultrasound, the position of the stone is identified. Under local anesthesia in adults and in general anesthesia in children, big intensity ultrasonic pulsation focused on the stone using gradual intensities is administered from the exterior. This procedure is repeated three times, sometimes it associates papillotomy for removal. An audiogram will be performed before and after the procedure, because it can affect the hearing. It is a gold standard in parotid sialolithiasis, but it is contraindicated in those with clotting disorders and in acute glandular inflammation ${ }^{21}$. Lithotripsy can also be intracorporeal, using endoscopic exploration of the duct and stone fragmentation with LASER. It is non-invasive, but expensive, it requires time and involved staff. The procedure does not prevent a relapse ${ }^{8,13,22}$.

\section{Viral infections of the salivary glands}

These infections can spread most frequently through hematogenous pathways, but ductal retrograde transmission is possible as well. Viral infections of the glandular parenchyma are not always clinically apparent. The viruses that spread from blood to the saliva without local signs are rabies, hepatitis, flu, poliomyelitis and those with local signs are mumps and AIDS $^{8}$.

1. Mumps appear especially in children, in the parotid gland and is often bilateral. It is determined by a very contagious paramyxovirus, it presents generalized infection and is endemic in communities. It spreads through direct contact with saliva or respiratory droplets from the mouth, nose, throat or urine. The incubation period is $16-18$ days, followed by a painful parotid swelling. The virus can get in the saliva after a week of glandular swelling, then ductal desquamation leads to secondary obstruction and expansion. Viremia signs like fever, headache, and arthralgia are gone in 3 to 7 days and the glandular swelling is gone in a few weeks ${ }^{23}$. Complications are local (suppurative parotitis) and general (meningoencephalitis, pancreatitis, orchitis, nephritis and neurosensory deafness). It opens the path to other infections. Laboratory tests show leucopenia, increase salivary amylase, hemagglutination, complement fixation tests and the histopathological test concludes the presence of acinar cells with cytoplasmic vacuolation. The treatment is established by the infectionist and it consists in isolation, rest, diet, hydration, vitamins and non-steroidal anti-inflammatory drugs ${ }^{13,24,25}$.

2. AIDS can manifest at the salivary glands level through lymphoproliferation or cysts and it leads to increased volume of major salivary glands and salivary dysfunction. It can appear in seropositive but also in seronegative patients with high risk of contracting HIV. Salivary disease is associated with $\mathrm{T}$ cell immunodeficiency and lymphocytosis. It usually affects two groups of age: children born of infected mothers and adults between 20 to 60 years of age ${ }^{26}$. Salivary signs could be the initial manifestation of HIV (often parotid signs). Bilateral cystic parotidomegaly appears in 15-30\% HIV children, usually in combination with lymphocytic interstitial pneumonia ${ }^{27}$. There is a soft, gradual increase of one or more salivary glands, often the parotid, dry mouth combined with dry eyes and arthralgia that can be mistaken with an autoimmune Sjögren syndrome. The glandular swelling is long lasting and stable. There are no autoantibodies present, but sometimes other opportunistic infections can appear. The biopsy reveals inflammatory infiltrates similar to Sjögren syndrome, epithelial cysts and intraglandular lymphomas. The studies have shown that salivary gland disease in AIDS is associated with a good prognosis ${ }^{28}$. The treatment is classic (oral hygiene, sialagogues, topical preparations with fluoride), volumetric regression with steroids and Zidovudine, surgical removal of nodular lesions with histopathological exam that can reveal lymphomas in more than $10 \%$ of HIV patients and rarely, parotid Kaposi sarcoma ${ }^{29}$. For monitoring these patients, on a larger scale, cytological puncture and CT/MRI are used. For 
abscess and suppurations, incision with draining and antibiotics are indicated ${ }^{8,27}$.

\section{Granulomatous infections of the salivary gland}

Affect the adjacent lymphatic system and the parotid gland. In fulminant cases, it affects the glandular parenchyma. Signs of the ailment can be moderate or gradual swelling of the ganglia may be present. It can be mistaken for a neoplastic tumor. The diseases that can lead to granulomatous infections are represented by TB, syphilis, actinomycosis, cat-scratch disease, toxoplasmosis and tularemia.

1. Cervicofacial TB is one of the rarest forms of extra-pulmonary TB. The incidence of extra-pulmonary TB has been on the rise since $1985^{30}$. The painless, enlarged glands are mimicking a slow growing neoplasm. Infection of the large salivary glands can be made through intraglandular ganglia and rarely, through infiltration of the parenchyma. The salivary implication can be primary (first infection) or secondary (resurgence of pulmonary TB). The parotid gland is affected in localized $\mathrm{TB}$ and the submandibular gland in systemic TB. Isolated implication of the salivary gland is typical for the parotid gland. There are two pathological types: localized, circumscribed ("cold abscess", intraparotid TB lymphadenitis) and diffuse (parotid hypertrophy, painless, irregularly shaped nodules of varying consistency). Both of them present enlargement of the adjacent lymph nodes and tendency to fistulize, leading to diagnostic difficulties. Under 50\% show a history of TB on a pulmonary $\mathrm{x}$-ray. Constitutional symptoms (fever, night sweats, weight loss) may be absent. Facial nerve affectation is rare. FNA may be negative in most of the cases. The diagnosis is histopathological. Sometimes, the diagnosis is established after the surgical removal of the gland. It requires an interdisciplinary pulmonology consultation. The treatment for salivary gland TB is typically through medication and is rarely surgical for intraparotid adenopathies that do not respond to treatment and for abscesses and fistulae resistant to treatment ${ }^{8,31}$. The non-TB mycobacterium disease usually appears in children between $1.5-3$ years. It is characterized by a rapid growing mass that lacks any signs of inflammation. Mycobacterium bovis is now infrequently involved due to milk pasteurization. The entry point is represented by the oral cavity, gums, lips, tonsils and it affects the parotid and the adjacent lymph nodes of the submandibular gland. Fistulization to the skin is frequent. Cutaneous tests are negative. Constitutional symptoms are absent. Pulmonary $\mathrm{x}$-ray is negative. Results of the bacterial culture is ready in 6 weeks. Diagnosis through FNA is preferable to incisional biopsy (the latter leads to refractory fistulas). Cutaneous tests with new tuberculin (Mycobacterium avium, malmoense, scrofulaceum) have been proven to be successful ${ }^{32}$. There is no evidence of person-to-person contagion. The treatment with anti-TB medication has proven to be inefficient. The surgical treatment spears the facial nerve ${ }^{13}$

2. Salivary gland syphilis rarely affects the salivary glands and mostly are tertiary lesions. Secondary lesions are rare. Tertiary syphilis parotitis can be diffuse (bilateral, gradual swelling, with firm and hard consistency), localized, named syphilitic gumma (early raw stage, softening, ulceration that lead to persistent fistulae) and pseudo tumoral (unilateral, fixed, hard consistency). Cervical lymphadenopathy is also present. The diagnosis is histopathological and serological. Treatment is only medical, on long-term and with large doses of penicillin ${ }^{33}$.

3. Actinomycosis is an infectious disease caused by Gram-positive anaerobic/facultative anaerobic bacteria. Actinomyces are commensal in the tonsils and dental cavities, they rarely infect the large salivary glands (parotid) in a retrograde, ductal manner. It affects the cervicofacial, ileocecal regions and the lungs. Actinomyces israeli is the most frequently encountered species followed by Actinomyces bovis and Actinomyces naeslundi. Poor dental hygiene and trauma to the mucosa allow the bacterial invasion. Diabetes, corticosteroid therapy and malnutrition are predisposing factors. In the primary form, the patient presents retrograde ductal infection, no pain, swollen salivary gland of firm consistency, slight pain on palpation of the salivary gland and excretion of saliva with specific yellow "sulfur like“ granules. The diagnosis is established by anaerobic bacterial cultures from the salivary secretion. In the secondary form, the patient presents altered, infiltrated, rigid skin, lesions of different stages (nodules, abscesses, fistulas) and „sprinkler skin“. The diagnosis is established by histopathological examination of the tissue. The treatment in actinomycosis of the salivary glands in the main form consists in antibiotics (penicillin is preferred, clindamycin, doxycycline, erythromycin), parenteral six weeks and then six months by oral administration. In the secondary form, surgery (incision with drainage, necrectomy) and antibiotics are recommended.

4. Cat-scratch disease is also a granulomatous lymphadenitis with cutaneous inoculation from domestic cat bites or claw scratches. More than $90 \%$ of the patients present exposure to cat scratches and $75 \%$ of the patients present exposure to cat scratches and dog bites. Dogs are implicated in 
$5 \%$ of the cases ${ }^{34}$. In USA, almost 12.000 people were hospitalized per year due to bartonellosis ${ }^{35}$. The pathogenic agent is a Gram-negative bacteria named Bartonella henselae. At the spot of inoculation, papules and pustules appear in 5 to 15 days from incubation ${ }^{36}$. At 3 weeks from incubation, the patient presents cervical lymphadenitis. The periglandular and intraglandular ganglia are affected. Suppurative phenomena occurs. The patient presents general symptoms such as fever and malaise and in rare cases, transient facial palsy is present. The diagnosis is established based on a history of contact with an animal (cat or dog that led to skin or eye lesions), local lymphadenopathy that occurred approximately 2 weeks after contact with the animal (negative bacterial cultures for other etiologies taken from the ganglia) and cutaneous test for the Cat-scratch disease (positive Hanger-Rose test). A positive histopathological exam for cat-scratch disease shows that the bacteria is present with the Warthin-Starry stain, granulomas, necrosis and micro-abscesses. Lymph node biopsy should be reserved for atypical cases. Recent serologic tests for Bartonella henselae antibodies are available. FNA of the lymph nodes is helpful in establishing a diagnosis. Treatment of cat-scratch disease is not necessary in the majority of cases, self-limiting lymph nodes heal spontaneously (2 to 4 months $)^{37}$. FNA of lymph nodes is also helpful for therapy. In case of immunosuppression, ciprofloxacin in adults and rifampicin in children are used $^{13}$.

5. Toxoplasmosis is a rare disease. The etiologic agent is Toxoplasma gondii. The usual host is the domestic cat. It is present in the parotid gland, intra- and parotid adjacent lymph nodes. Toxoplasma trophozoites and cysts enter the human body by ingestion of contaminated meat (lamb, cow, chicken), rarely they enter the body by ingestion of cysts from the cat's fecal matter. Digestion of the cyst's capsule leads to a multiplication of the trophozoites and hematogenous dissemination in the reticular organs. It has two forms: lymphadenopathic form (common, isolated cervical adenopathy, histopathologic exam and serologic tests confirm the diagnosis) and disseminated form (found in immunosuppressed individuals, symptoms include muscle pain, lethargy, anorexia, hepatomegaly, splenomegaly, pericarditis, myocarditis ${ }^{38}$. The treatment for toxoplasmosis consists in administration of pyrimethamine and trisulfapyrimidine (done by an infectionist or parasitologist).

6. Tularemia is determined by a Gram-negative bacterium, Franciscella tularensis. The human transmission is possible by tick bite, handling of contaminated meat or contaminated aerosols. The patient presents a red papule at the entry point (which can be in the parotid region). The incubation period lasts 2 to 10 days in which nearby lymph nodes get swollen, tense and become painful. Fever, headaches and other toxic signs appear. If the infection is gained through the digestive or respiratory tract, there are no cutaneous manifestations and diffuse lymphadenopathies are present ${ }^{39}$. The microorganism stays viable inside the cell, which can lead to chronic granulomatous disease. Franciscella tularensis is rarely isolated from human blood. Serological tests are required. Aggressive lymph node palpation is not recommended because it can lead to dissemination. The treatment is successful if erythromycin, tetracycline or ciprofloxacin are used. The pathogen is resistant to most $\beta$-lactamases and penicillins. The fluctuating cysts will be incised and drained after antibiotics administration and the remission of the systemic symptomatology ${ }^{1}$.

\section{Conclusions}

Inflammation of the salivary glands manifest differently, depending on the etiologic agent and patient. Acute suppurative infections are bacterial in origin, have a recent onset and usually affect debilitated patients. Chronic sialadenitis has recurring episodes of pain and swelling and is found in adults. Viral systemic infections (mumps, AIDS) can cause localized salivary pathology at any age, but have different clinical manifestations. Granulomatous infection of the salivary glands can affect children but also old people and often lead to a gradual increase in the size of the salivary gland and lymph nodes and it can be mistaken for a neoplastic tumor ${ }^{40}$. Differential diagnosis is based on a carefully done anamnesis, on the physical examination and identifying the etiologic agent that can cause the disease. The ENT specialist can establish a precise diagnosis and recommend a good treatment. Inflammatory and infectious pathology of the salivary glands is multifactorial and has a multimodal therapy ${ }^{41}$. Surgical treatment is mandatory in case of abscess. Suppurations of the salivary glands in immunosuppressed individuals can extend to cervicofacial regions and the mediastinum and require immediate treatment. Antibiotics are compulsory and are usually recommended by the infectionist or parasitologist. Interdisciplinary collaboration with the infectionist, parasitologist and radiologist is the golden standard towards the benefit of the patient. A careful review of the case also prevents possible facial and lingual nerve damage, with legal consequences. 
Early presentation of the patient guarantees the therapeutic success.

\section{Compliance with Ethics Requirements:}

„The authors declare no conflict of interest regarding this article"

\section{RefEREnCES}

1. Cumming C, Fredrickson J, Harker L, Krause C, Richardson M, Schuller D. Otolaryngology Head and Neck Surgery. 3rd ed.: Mosby; 2010.

2. Sasaki C. Sialadenitis. Cited 2019 October 23. Available from: https://www.merckmanuals.com/professional/ ear-nose-and-throat-disorders/oral-and-pharyngeal-disorders/sialadenitis.

3. Fattahi T, Lyu P, Sickels J. Management of acute suppurative parotitis. Journal of Oral and Maxillofacial Surgery. 2002; 60(4):446-448.

4. Brook I. The bacteriology of salivary gland infections. Oral and Maxillofacial Surgery Clinics of North America. 2009; 21(3):269-274.

5. Bucur A, Dinca O. Patologia glandelor salivare. Cited 2019 October 23. Available from: https://www.academia. edu/37333777/13._Patologia_glandelor_salivare.pdf.

6. Avery C. BMJ Best Practice. 2019. Cited 2019 October 23. Available from: https://bestpractice.bmj.com/topics/ en-gb/1038.

7. Spiegel R, Miron D, Sakran W, Horovitz Y. Acute neonatal suppurative parotitis: Case reports and review. The Pediatric Infectious Disease Journal. 2004; 23(1):76-78.

8. Bucur A, Navarro Vila C, Lowry J, Acero J. Compendiu de Chirurgie Oro-Maxilo-Faciala Bucharest: Q. Med Publishing; 2009.

9. Razek AAKA, Mukherji S. Imaging of sialadenitis. The Neuroradiology Journal. 2017; 30(3):205-215.

10. Tucci F, Roma R, Bianchi A, De Vincentiis G, Bianchi P. Juvenile recurrent parotitis: Diagnostic and therapy effectiveness of sialography. Retrospective study on 110 children. International Journal of Pediatric Otorhinolaryngology. 2019;124:179-184.

11. Ugga L, Ravanelli M, Pallottino A, Farina D, Maroldi R. Diagnostic work-up in obstructive and inflammatory salivary gland disorders. ACTA Otorhinolaryngologica Italica. 2017;37(2):83-93.

12. Wang S, Marchal F, Zou Z, Zhou J, Qi S. Classification and management of chronic sialadenitis of the. Journal of Ora Rehabilitation. 2009; 36(1):2-8.

13. Anniko M, Bernal-Sprekelsen M, Bonkowsky V, Bradley P, Iurato S. Otorhinolaryngology, Head and Neck Surgery. 1st ed. Germany: Springer Verlag; 2010.

14. Capaccio P, Torretta S, Ottaviani F, Sambataro G, Pignataro L. Modern management of obstructive salivary diseases. ACTA Otorhinolaryngologica Italica. 2007; 27(4):161-172.

15. Bodner L. Salivary gland calculi: Diagnostic imaging and surgical management. Compendium of continuing education in dentistry. 1993;14:572-586.

16. Ledesma-Montes C, Garcés-Ortíz M, Salcido-García J, Hernández-Flores F, Hernández-Guerrero J. Giant sialolith: case report and review of the literature. Journal of Oral and Maxillofacial Surgery, 2007;65(1):128-130.
17. Bodner L. Giant salivary gland calculi: diagnostic imaging and surgical management. Oral Surgery, Oral Medicine, Oral Pathology, Oral Radiology, and Endodontology. 2002; 94(3):320-323.

18. Bates D, O'Brien C, Tikaram K, Painter D. Parotid and submandibular sialadenitis treated by salivary gland excision. The Australian and New Zealand Journal of Surgery. 1998; 68(2): 120-124.

19. Hald J, Andreassen K. Submandibular gland excision: shortand long-term complications. ORL. 1994; 56(2):87-91.

20. Nahlieli O, Shacham R, Yoffe B, Eliav E. Diagnosis and treatment of strictures and kinks in salivary gland ducts. Journal of Oral and Maxillofacial Surgery: official journal of the American Association of Oral and Maxillofacial Surgeons. 2001; 59(5):484-490.

21. Eggers G, Chilla R. Ultrasound guided lithotripsy of salivary calculi using an electromagnetic lithotriptor. International Journal of Oral and Maxillofacial Surgery. 2005; 34(8):890-894.

22. Koch M, Hung S, Su C, Lee K, Iro H, Mansopoulos K. Intraductal lithotripsy in sialothitiasis with two different HO: YAG lasers: presenting parameters, effectiveness, success, rates. European Review for Medical and Pharmacological Sciences. 2019; 23(13):5548-5557.

23. McQuone S. Acute viral and bacterial infections of the salivary glands. Otolaryngologic Clinics of North America. 1999; 32(5):793-811.

24. Barskey A, Schulte C, Rosen J, et al. Mumps outbreak in Orthodox Jewish communities in the United States. The New England Journal of Medicine. 2012;367(18):1704-1713.

25. Rusu C, Vaculin I, Cevotanu D. Particularitatile clinico-evolutive ale oreionului la militarii in termen. Revista de Medicina Militara. 2013;2(10):74-76.

26. Jeffers L, Webster-Cyriaque J. Viruses and salivary gland disease (SGD). Advances in Dental Research. 2011; 23(1):79-83.

27. Nizamuddin I, Koulen P, McArthur C. Contribution of HIV infection, AIDS, and antiretroviral therapy to exocrine pathogenesis in salivary and lacrimal glands. International Journal of Molecular Sciences. 2018;19(9):2747.

28. Sekikawa Y, Hongo I. HIV-associated benign lymphoepithelial cysts of the parotid glands confirmed by HIV-1 p24 antigen immunostaining. BMJ Case Rep 2017;2017: pii:bcr2017221869.

29. Reznik D. Oral manifestations of HIV disease. Topics in HIV medicine: a publication of the International AIDS Society, USA. 2005/2006;13(5):143-148.

30. Adada H, Valley M, Nour S, et al. Epidemiology of extra-pulmonary tuberculosis in the United States: high rates persist in the post-HIV era. The International Journal of Tuberculosis and Lung Disease. 2014;18(12):1516-1521.

31. Virmani N, Dabholkan J. Primary tubercular sialadenitis a diagnostic dillema. Iranian Journal of Otorhinolaryngology. 2019;31(102):45-50.

32. Cox H, Brightwell A, Riordan T. Non-tuberculous mycobacterial infections presenting as salivary gland masses in children: investigation and conservative management. The Journal of Laryngology and Otology. 1995;109(6):525-530.

33. Hira S, Hira R. Parotitis with secondary syphilis: a case report. The British Journal of Venereal Diseases. 1984;60(2):121-122.

34. Álvarez-Fernández A, Breitschwerdt E, Solano-Gallego L. Bartonella infections in cats and dogs including zoonotic aspects. Parasites $\mathcal{E}$ Vectors. 2018;11(1):624.

35. Nelson C, Saha S, Mead P. Cat-Scratch Disease in the United States, 2005-2013. Emerging Infectious Diseases. 2016;22(10):1741-1746. 
36. Mazur-Melewska K, Mania A, Kemnitz P, Figlerowicz M, Służewski W. Cat-scratch disease: a wide spectrum of clinical pictures. Postępy Dermatologii i Alergologii. 2015;32(3):216-220.

37. Klotz S, Ianas V, Elliott S. Cat-scratch Disease. American Family Physician. 2011; 83(2):152-155.

38. Tiglis M, Neagu TP, Elfara M, et al. Nefopam and its role in modulating acute and chronic pain. Rev Chim (Bucharest) 2018;69(10):2877-2880
39. Diaconu C, Balaceanu A, Morosan E. Sepsis biomarkers: past, present and future. Farmacia 2015;63(6):811-815.

40. Diaconu C, Balaceanu A, Ghinescu M. A neck mass that disapears at compression: is it a reason for concern? Acta Medica Mediterranea 2015;31(2):339-341.

41. Tica OA, Tica O, Antal L, et al. Modern oral anticoagulant treatment in patients with atrial fibrillation and heart failure: insights from the clinical practice. Farmacia 2018;66(6):972-976. 